\title{
Dissipative time-dependent quantum transport theory
}

\author{
Yu Zhang, Chi Yung Yam, and GuanHua Chen ${ }^{\text {a) }}$ \\ Department of Chemistry, The University of Hong Kong, Hong Kong
}

(Received 8 February 2013; accepted 8 April 2013; published online 26 April 2013)

\begin{abstract}
A dissipative time-dependent quantum transport theory is developed to treat the transient current through molecular or nanoscopic devices in presence of electron-phonon interaction. The dissipation via phonon is taken into account by introducing a self-energy for the electron-phonon coupling in addition to the self-energy caused by the electrodes. Based on this, a numerical method is proposed. For practical implementation, the lowest order expansion is employed for the weak electronphonon coupling case and the wide-band limit approximation is adopted for device and electrodes coupling. The corresponding hierarchical equation of motion is derived, which leads to an efficient and accurate time-dependent treatment of inelastic effect on transport for the weak electron-phonon interaction. The resulting method is applied to a one-level model system and a gold wire described by tight-binding model to demonstrate its validity and the importance of electron-phonon interaction for the quantum transport. As it is based on the effective single-electron model, the method can be readily extended to time-dependent density functional theory. ( 2013 AIP Publishing LLC. [http://dx.doi.org/10.1063/1.4802592]
\end{abstract}

\section{INTRODUCTION}

Time-dependent quantum transport is an important subject of research. ${ }^{1-9}$ The existence of rigorous first-principles method for open electronic system was proved based on the time-dependent holographic electron density theorem (TDHEDT) ${ }^{3,10}$ As an extension of Runge-Gross theorem ${ }^{11}$ to open system, TD-HEDT established one-to-one mapping between electron density of reduced system and time-dependent external potential, thus laid the foundation of time-dependent density functional theory (TDDFT) for time-dependent quantum transport. Consequently, a practical scheme to study the time-dependent quantum transport was developed by the combination of TDDFT and non-equilibrium Green's function (NEGF) method, which was termed as TDDFT-NEGF and has been applied to study current response to ac bias voltage. ${ }^{12}$ However, the TDDFT-NEGF method employs the adiabatic wide-band limit (AWBL) approximation to simplify the dissipation functional, ${ }^{3}$ which limits its application to low and slow-varying bias. In order to improve the performance of TDDFT-NEGF, a hierarchical equation of motion (HEOM) was proposed to solve the dissipation functional by the insight from recent progress in HEOM approach of quantum dissipation theory. ${ }^{13}$ The resulting TDDFT-NEGF-HEOM is an exact formalism for the time-dependent quantum transport within the TDDFT framework, ${ }^{4,6}$ which can be applied to both finite and zero temperature systems. In addition, TDDFT-NEGFHEOM is able to go beyond the AWBL approximation used in Ref. 3.

However, previous studies in the TDDFT for quantum transport did not take the dissipation due to electron-phonon interaction (EPI) into account. In the presence of phonon, electron has the probability of being scattered off inelastically by phonon. Inelastic scattering of transport electron and

\footnotetext{
a)Electronic mail: ghc@everest.hku.hk
}

energy dissipation play a vital role in device characteristics, working performance, and stability. Its effects in the single molecule junction have attracted a lot of attention both experimentally and theoretically. ${ }^{14-26}$ There are existing models to deal with inelastic scattering due to EPI in molecular junctions, like Fermi's golden rule (FGR), ${ }^{27}$ self-consistent Born approximation (SCBA) with NEGF method. ${ }^{28}$ SCBA has demonstrated good agreement with exact scattering theory ${ }^{29}$ in the weak coupling limit. However, the expensive computational cost of SCBA not only limits the system size of static simulation, but also renders time-dependent studies intractable. A reasonable approximation is important for practical purpose. The lowest order expansion (LOE) has been proposed to reduce the computational cost of SCBA. ${ }^{16-19}$ It simplifies the SCBA at the conditions: (i) EPI is weak; (ii) density of states (DOS) of the leads and device are slowly varying over a few phonon-energies around the Fermi energy $\epsilon_{f}$, i.e., they assumed energy-independent retarded Green's function $G_{0}^{r}(\epsilon) \approx G_{0}^{r}\left(\epsilon_{f}\right)$ and line-width function $\Lambda_{L / R}(\epsilon)$ $\approx \Lambda_{L / R}\left(\epsilon_{f}\right)$. It is shown that LOE simulations compare closely with those obtained with SCBA simulations. ${ }^{28}$

While the majority of studies on quantum transport with EPI focus on steady state, it is also important to study transient effects and response to dynamic external fields. In this work, TDDFT-NEGF-HEOM method is extended to include EPI's effect. This is achieved by including the self-energy contributed by EPI. Due to the computational cost of TDDFTNEGF-HEOM method, the WBL approximation for leads is adopted in this work (TDDFT-NEGF-HEOM-WBL). As DFT or TDDFT can be regarded as an effective single-electron model, the general NEGF-HEOM-WBL formalism will be developed in the framework of a single-electron model with EPI. For weak EPI case, the LOE is used, and corresponding NEGF-HEOM-WBL formalism is established. The LOE used in this work is slightly different from that in Refs. 16-19 where the non-interacting Green's function and line-width 
function are both regarded as energy independent. While the current method only assumes energy independent line-width function. To demonstrate the validity of the new formalism, the tight-binding model is adopted to simulate the transient currents through one-site model and a gold monoatomic chain.

The paper is organized as follows. Section II introduces the NEGF-HEOM-WBL formalism for time-dependent quantum transport with EPI from a single-electron Hamiltonian. Numerical studies and related discussion are given in Sec. III. Section IV summarizes the method developed in this work.

\section{METHODOLOGY}

Since DFT or TDDFT can be regarded as an effective single-electron model, we confine ourselves to the effective single-electron Hamiltonian as follows:

$$
H=H_{D}+\sum_{\alpha}\left[H_{\alpha}+H_{\alpha D}\right]
$$

where Hamiltonian for the device region is

$$
H_{D}=\sum_{\mu \nu} h_{\mu \nu} d_{\mu}^{\dagger} d_{\nu}+\sum_{q} \omega_{q} b_{q}^{\dagger} b_{q}+\sum_{q, \mu \nu} \gamma_{\mu \nu}^{q} d_{\mu}^{\dagger} d_{\nu} B_{q} .
$$

The EPI is taken into account in harmonic approximation, $d_{\mu}^{\dagger}$ and $d_{v}$ are the electronic creation and annihilation operators in the device region, respectively; and $h_{\mu \nu}$ is the corresponding single-electron Fock matrix. $B_{q}=\left(b_{q}^{\dagger}+b_{q}\right)$, where $b_{q}^{\dagger}$ and $b_{q}$ are phonon creation and annihilation operators, respectively. $\omega_{q}$ and $\gamma_{\mu \nu}^{q}$ are phonon frequency and electron-phonon coupling constant, respectively. Phonon modes in leads should in principle be considered, as they have effects on the phonon modes of device region. However, phonon modes are only assumed to exist in device region for simplification. Their coupling with phonon modes in leads can be considered in a phenomenological way, for instance, introducing a rate equation for the phonon occupation number which accounts for the coupling. The Hamiltonian of lead $\alpha$ is $H_{\alpha}=\sum_{k} \epsilon_{k_{\alpha}} c_{k_{\alpha}}^{\dagger} c_{k_{\alpha}}$ and the Hamiltonian for the coupling between device and lead $\alpha$ is $H_{\alpha D}=\sum_{k_{\alpha}, \mu}\left(V_{k_{\alpha} \mu} c_{k_{\alpha}}^{\dagger} d_{\mu}+H . c.\right) . c_{k_{\alpha}}^{\dagger}$ and $c_{k_{\alpha}}$ are the electronic creation and annihilation operators in the lead $\alpha$, respectively; $V_{k_{\alpha} \mu}$ is the device-lead coupling strength.

\section{A. NEGF-HEOM-WBL scheme without EPI}

NEGF-HEOM is an exact formalism for non-interacting systems. ${ }^{4}$ In order to reduce the computational cost and apply it on large systems, WBL approximation is usually adopted. WBL assumes infinite and energy independent band-width. The resulting NEGF-HEOM-WBL scheme provides an efficient way to simulate the realistic systems. ${ }^{4,5}$ In absence of EPI, the equation of motion (EOM) of reduced single electron density matrix (RSDM) is

$$
i \dot{\sigma}(t)=[h(t), \sigma(t)]-\sum_{\alpha}\left[\varphi_{\alpha}(t)-\varphi_{\alpha}^{\dagger}(t)\right]
$$

where the auxiliary density matrix $\varphi_{\alpha}(t)$ is

$$
\varphi_{\alpha}(t)=i \int_{-\infty}^{t} d \tau\left[G^{<}(t, \tau) \Sigma_{\alpha}^{>}(\tau, t)-G^{>}(t, \tau) \Sigma_{\alpha}^{<}(\tau, t)\right],
$$

where $\alpha=L, R . G^{<}$and $G^{>}$are the lesser and greater Green's functions of device, and $\Sigma_{\alpha}^{<}$and $\Sigma_{\alpha}^{>}$are the lesser and greater self-energies due to the lead $\alpha$, respectively. The first term of Eq. (4) is interpreted as the outcoming rate of electron from device to lead $\alpha$. While the second term of Eq. (4) is interpreted as the incoming rate of electron from lead $\alpha$ to device. Hence, $\varphi_{\alpha}(t)$ corresponds to the net rate of electron going through the interface between lead $\alpha$ and device. Hence, transient current can be evaluated by taking the trace of the auxiliary density matrix,

$$
I_{\alpha}(t)=i \operatorname{Tr}\left[\varphi_{\alpha}(t)-\varphi_{\alpha}^{\dagger}(t)\right] .
$$

Within the WBL approximation, the finite-temperature selfenergy is

$$
\Sigma_{\alpha}^{<,>}(\tau, t)= \pm 2 i \int d \epsilon f_{\alpha}^{ \pm}(\epsilon) e^{i \int_{\tau}^{t}\left[\epsilon+\Delta\left(t_{1}\right)\right] d t_{1}} \Lambda_{\alpha},
$$

where $\quad f_{\alpha}^{ \pm}(\epsilon)=1 /\left(e^{ \pm \beta\left(\epsilon-\mu_{\alpha}\right)}+1\right) . \quad \Lambda_{\alpha}=\pi \sum_{k_{\alpha}}|V|^{2}$ $\delta\left(\epsilon_{f}-\epsilon_{k_{\alpha}}\right)$ is the line-width function due to the lead $\alpha$ at Fermi energy $\epsilon_{f}$. Applying the Padé approximation, Fermi distribution function is expanded as ${ }^{30}$

$$
f_{\alpha}^{ \pm}(\epsilon) \approx \frac{1}{2} \mp \sum_{k}^{N} \frac{1}{\beta} \frac{2 \eta_{k}\left(\epsilon-\mu_{\alpha}\right)}{\left(\epsilon-\mu_{\alpha}\right)^{2}+\left(\zeta_{k} / \beta\right)^{2}},
$$

where $\pm i \zeta_{k} / \beta+\mu_{\alpha}$ are the $k$ th Padé poles in the upper and lower half plane, respectively; $\eta_{k} / \beta$ is the corresponding coefficient. Hence, the integration in Eq. (6) can be obtained analytically through contour integral and residue theorem, the resulting expression of self-energy is

$$
\Sigma_{\alpha}^{<,>}(\tau, t) \approx \pm \frac{1}{2} i \delta(t-\tau) \Lambda_{\alpha}+x \sum_{k} \Sigma_{\alpha, k}^{x}(\tau, t),
$$

where $x=+$ for $t \geq \tau$ and $x=-$ for $t<\tau$. The sign $x$ corresponds to upper $(+)$ and lower half plane $(-)$ contour integration. $\Sigma_{\alpha k}^{ \pm}(\tau, t)$ is defined as $\Sigma_{\alpha k}^{ \pm}(\tau, t)=\frac{2}{\beta} \eta_{k} e^{i \int_{\tau}^{t} \epsilon_{\alpha k}^{ \pm}\left(t_{1}\right) d t_{1}} \Lambda_{\alpha}$, $\epsilon_{\alpha k}^{ \pm}(t)= \pm i \zeta_{k} / \beta+\mu_{\alpha}+\Delta_{\alpha}(t)$. Based on the approximation on self-energy described in Eq. (8), the auxiliary density matrix is rewritten as

$$
\varphi_{\alpha}(t)=i[\sigma(t)-1 / 2] \Lambda_{\alpha}+\sum_{k} \tilde{\varphi}_{\alpha k}(t)
$$

The first term on the right hand side (RHS) of Eq. (9) comes from the integration over lesser/greater Green's function and delta function, and

$$
\tilde{\varphi}_{\alpha k}(t)=-i \int_{-\infty}^{\infty} d \tau G^{r}(t, \tau) \Sigma_{\alpha k}^{+}(\tau, t)
$$

is the component of first-tier auxiliary density matrix, which is evaluated through its EOM. Within WBL, time derivatives of $G^{r}(t, \tau)$ and $\Sigma_{\alpha k}^{+}(\tau, t)$ are linear equations of themselves. It is straightforward to write down the EOM of the first-tier auxiliary density matrix as

$$
i \dot{\tilde{\varphi}}_{\alpha k}(t)=-i \frac{2}{\beta} \eta_{k} \Lambda_{\alpha}-\left[G^{r}\left(\epsilon_{\alpha k}^{+}\right)\right]^{-1} \tilde{\varphi}_{\alpha k}(t),
$$


where $\left[G^{r}\left(\epsilon_{\alpha k}^{+}(t)\right)\right]^{-1}$ is defined as $\left[G^{r}\left(\epsilon_{\alpha k}^{+}(t)\right)\right]^{-1}=\left[\epsilon_{\alpha k}^{+}(t)\right.$ $-h(t)+i \Lambda]$, and $\Lambda=\sum_{\alpha} \Lambda_{\alpha}$ is the total line-width function. Hence, Eqs. (3), (9), and (11) constitute a practical and efficient scheme for simulation of transient dynamics of noninteracting system. The number of auxiliary density matrices is determined by the truncation of Padé expansion. The EOMs can be numerically solved by employing the fourth order Runge-Kutta method with corresponding initial conditions. Transient current at each time step can be obtained by solving Eq. (5).

\section{B. NEGF-HEOM-WBL scheme with EPI}

\section{Exact EOM of RSDM with EPI}

We consider transport electrons as a system interacting with phonon in the device region. The EPI can be treated by the standard perturbation theory on the Keldysh contour. Starting from the electron Green's function $G_{0}\left(\tau, \tau^{\prime}\right)$ without EPI (the electronic coupling between device and lead has been considered), the self-energy to electron caused by EPI, $\Sigma_{e p}\left(\tau, \tau^{\prime}\right)$, can be obtained through the perturbation theory by summing over the Feynman diagrams into infinite order. The resulting electron Green's function is given by the Dyson equation (A simplified notation $\left[G_{0} \Sigma_{e p} G\right]\left(\tau, \tau^{\prime}\right)$ is used instead of $\int d \tau_{1} d \tau_{2} G_{0}\left(\tau, \tau_{1}\right) \Sigma\left(\tau_{1}, \tau_{2}\right) G\left(\tau_{2}, \tau^{\prime}\right)$.),

$$
G\left(\tau, \tau^{\prime}\right)=G_{0}\left(\tau, \tau^{\prime}\right)+\left[G_{0} \Sigma_{e p} G\right]\left(\tau, \tau^{\prime}\right),
$$

where the self-energy $\Sigma_{e p}\left(\tau, \tau^{\prime}\right)$ is the functional of phonon Green's function $D\left(\tau, \tau^{\prime}\right)$ and electron Green's function $G\left(\tau, \tau^{\prime}\right)$. The self-energy to electron caused by EPI contains Hartree component and Fock component with vertex part as shown in Figs. 1(a) and 1(b), ${ }^{31-33}$ then the self-energy is expressed as

$$
\begin{aligned}
\Sigma_{e p}\left(\tau, \tau^{\prime}\right)= & i \sum_{q} \gamma_{q}\left[\delta\left(\tau, \tau^{\prime}\right) \int d \tau_{1} D\left(\tau, \tau_{1}\right) G\left(\tau_{1}, \tau_{1}\right)\right. \\
& \left.+\int d \tau_{1} \int d \tau_{2} D\left(\tau, \tau_{1}\right) G\left(\tau, \tau_{2}\right) \Gamma\left(\tau_{2}, \tau_{1} ; \tau^{\prime}\right)\right] \gamma_{q},
\end{aligned}
$$

where $D\left(\tau, \tau^{\prime}\right)$ is the dressed phonon Green's function and $\Gamma\left(\tau_{2}, \tau_{1} ; \tau^{\prime}\right)$ is the vertex part as shown in Fig. 2, detail discussions about vertex part can be found in Refs. 31 and 33 . The first term and second term on the RHS of Eq. (13) represents the Fock component with vertex part and Hartree component, respectively. The vertex part $\Gamma\left(\tau_{2}, \tau_{1} ; \tau^{\prime}\right)$ contains infinite number of irreducible Feynman diagrams, its expression is not given explicitly here since it is nontrivial to write down an exact expression for this part. Similar to electron Green's function, phonon Green's function can also be evaluated through the Dyson equation,

$$
D\left(\tau, \tau^{\prime}\right)=d_{0}\left(\tau, \tau^{\prime}\right)+\left[d_{0} \Pi D\right]\left(\tau, \tau^{\prime}\right),
$$

where $d_{0}\left(\tau, \tau^{\prime}\right)$ is bare phonon Green's function, $\Pi\left(\tau, \tau^{\prime}\right)$ is the self-energy to phonon caused by the interaction with electron, its expression is not considered here. Equations (12)(14) provide a self-consistent scheme for the evaluation of
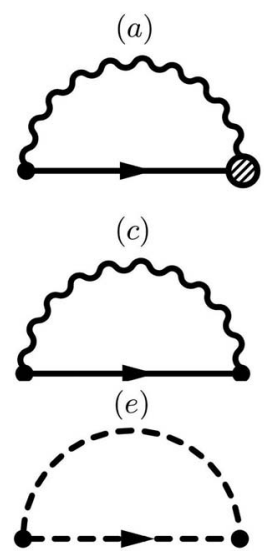

(b)

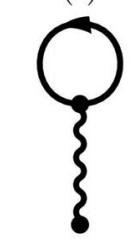

$(d)$

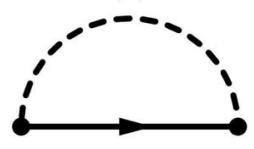

FIG. 1. Feynman diagram presentation of self-energy to electron caused by EPI. The arrow is dressed electron Green's function; the wiggly line is dressed phonon Green's function; the dashed line is bare phonon Green's function and the dashed arrow is bare electron Green's function; the blob is vertex part. (a) The Fock component with vertex part; (b) the Hartree component; (c) the Fock component within SCBA scheme; (d) similar to (c) except the phonon Green's function is the bare one; and (e) the LOE scheme, both phonon and electron Green's function are the bare ones.

electron and phonon Green's functions through Dyson equation in terms of the corresponding self-energies which are dependent on electron and phonon Green's functions. The EOM of RSDM can be derived through the time derivative of lesser electron Green's function which is obtained by projecting the $G\left(\tau, \tau^{\prime}\right)$ on to real-time axis. The resulting EOM of RSDM reads

$$
i \dot{\sigma}(t)=[h(t), \sigma(t)]-\sum_{\alpha}\left[\varphi_{\alpha}(t)-\varphi_{\alpha}^{\dagger}(t)\right],
$$

where $\alpha=L, R, e p$. Equation (15) has the same form as Eq. (3). The difference lies in the inclusion of $\varphi_{e p}(t)$ which arises from EPI. The expression of auxiliary density matrix $\varphi_{\alpha}(t)$ is

$$
\varphi_{\alpha}(t)=i \int_{-\infty}^{t} d \tau\left[G^{<}(t, \tau) \Sigma_{\alpha}^{>}(\tau, t)-G^{>}(t, \tau) \Sigma_{\alpha}^{<}(\tau, t)\right]
$$

Compare to Eq. (4), Green's function in Eq. (16) contains the dissipative effect due to EPI. $\varphi_{L / R}(t)$ represents the interaction between electron of device and that of leads; $\varphi_{e p}(t)$ describes the dissipative process of electron in presence of EPI. And Eq. (15) is the EOM for RSDM matrix of the open electronic device interacting with both electron in leads and phonon in device. Considering $\varphi_{\alpha}(t)$ can be determined exactly, Eq. (15) gives exact solution to density matrix and therefore any physical quantities.

In order to ensure the particle conservation or current continuity, $\operatorname{Tr}\left[\varphi_{e p}(t)-\varphi_{e p}^{\dagger}(t)\right]$ has to be zero since there is no particle dissipation due to EPI. Exact self-energy, i.e.,

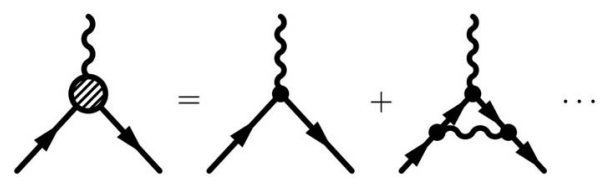

FIG. 2. Feynman diagram presentation of vertex part. 
Eq. (13), guarantees this fundamental conservation law. Actually, Eq. (13) can be written as the functional derivative of $\Phi$-derivable functional:

$$
\Sigma_{e p}[G]\left(\tau, \tau^{\prime}\right)=\frac{\delta \Phi[G]}{\delta G\left(\tau^{\prime}, \tau\right)} .
$$

The exact $\Phi[G]$ functional can be obtained by summing over all skeleton diagrams, i.e., all closed diagrams as described by Luttinger and Ward. ${ }^{34,35}$ As shown by Baym and Kadanoff, ${ }^{36,37}$ self-energy ensures the conservation law when it can be written as the functional derivative of $\Phi$-derivable functional. For an open system, conservation law implies the current continuity, i.e., the time-derivative of density is equal to the sum of currents flowing in and out of the system. The validity of the conservation law for $\Phi$-derivable functional is guaranteed by the invariance of $\Phi$ functional under gauge transformations. If the Hamiltonian of system is changed by an arbitrary gauge function $\Xi$, Green's function becomes

$$
G[\Xi]_{i j}\left(\tau, \tau^{\prime}\right)=e^{-i \Xi_{i}(\tau)} G_{i j} e^{i \Xi_{j}\left(\tau^{\prime}\right)} .
$$

Since $\Phi$ functional is made up of all closed diagrams in terms of Green's function $G\left(\tau, \tau^{\prime}\right)$, the exponential factors cancel at each vertex, i.e., $\Phi[G]$ functional is gauge invariance. Therefore,

$$
\begin{aligned}
0 & =\frac{\delta \Phi[G]}{\delta \Xi\left(\tau_{1}\right)}=\int d \tau d \tau^{\prime}\left[\frac{\Phi[G]}{\delta G\left(\tau^{\prime}, \tau\right)}\right] \frac{\delta G\left(\tau^{\prime}, \tau\right)}{\delta \Xi\left(\tau_{1}\right)} \\
& =\int d \tau d \tau^{\prime} \Sigma_{e p}\left(\tau, \tau^{\prime}\right) \frac{\delta G\left(\tau^{\prime}, \tau\right)}{\delta \Xi\left(\tau_{1}\right)},
\end{aligned}
$$

inserting the derivative of Green's function with respect to $\Xi\left(\tau_{1}\right)$ from Eqs. (17) to (18) gives

$$
\begin{aligned}
0= & i \sum_{i j} \int d \tau d \tau^{\prime}\left[\Sigma_{e p, i j}\left(\tau, \tau^{\prime}\right) G_{j i}\left(\tau^{\prime}, \tau\right)\right. \\
& \left.-G\left(\tau, \tau^{\prime}\right)_{i j} \Sigma_{e p, j i}\left(\tau^{\prime}, \tau\right)\right] \Xi_{i}(\tau) .
\end{aligned}
$$

Since it is true for arbitrary $\Xi$, it ends up with

$$
\operatorname{Tr}\left\{i \int d \tau^{\prime}\left[\Sigma_{e p}\left(\tau, \tau^{\prime}\right) G\left(\tau^{\prime}, \tau\right)-G\left(\tau, \tau^{\prime}\right) \Sigma_{e p}\left(\tau^{\prime}, \tau\right)\right]\right\}=0 .
$$

Projecting Eq. (20) on real-time axis gives $\operatorname{Tr}\left[\varphi_{e p}(t)\right.$ $-\varphi_{e p}^{\dagger}(t)=0$, i.e., particle conservation or current continuity is satisfied whenever self-energy can be written as the functional derivative of $\Phi$-derivable functional.

\section{EOM of RSDM with EPI within the scheme of SCBA}

Even though Eq. (13) provides a systematic way for the evaluation of self-energy to electron caused by EPI, it is impractical to sum over the Feynman diagrams in the vertex part into infinite order. One approach is to truncate the vertex part to the first order, $\Gamma\left(\tau_{2}, \tau_{1} ; \tau^{\prime}\right) \approx \delta\left(t_{2}, \tau^{\prime}\right) \delta\left(\tau_{1}, \tau^{\prime}\right)$, the selfenergy becomes

$$
\begin{aligned}
\Sigma\left(\tau, \tau^{\prime}\right)= & i \sum_{q} \gamma_{q}\left[D\left(\tau, \tau^{\prime}\right) G\left(\tau, \tau^{\prime}\right)\right. \\
& \left.+\delta\left(\tau, \tau^{\prime}\right) \int d \tau_{1} D\left(\tau, \tau_{1}\right) G\left(\tau_{1}, \tau_{1}\right)\right] \gamma_{q}
\end{aligned}
$$

This is the widely used SCBA scheme. Since the second term on the RHS of Eq. (21) only contributes to the energy shift of electron, it is usually ignored. ${ }^{28,38}$ The resulting self-energy is represented as Fig. 1(c). In principle, both electron and phonon are non-equilibrium and are dressed by EPI. However, since characteristic time scale of electronic process is much smaller than that of phonon process, EPI dissipative of phonon is ignored for simplification, i.e., phonon is assumed in equilibrium and unperturbated by electron. Therefore, the EPI dressed electron Green's function $G(t, \tau)$ and bare phonon Green's function $d_{0}(t, \tau)$ are used to construct the self-energy $\Sigma_{e p}(t, \tau)$ as shown in Fig. 1(d). The bare lesser phonon Green's function is $d_{q}^{0,<}(t, \tau)$ $=-i \sum_{ \pm} N_{q}^{ \pm} e^{ \pm i \omega_{q}(t-\tau)}$, here $N_{q}^{ \pm}=N_{q}+\frac{1}{2} \pm \frac{1}{2} . N_{q}$ and $\omega_{q}$ are occupation number of phonon and phonon frequency for the $q$ th mode, respectively. Hence, the interacting self-energy is $^{39}$

$$
\Sigma_{e p}^{<}\left(t_{1}, t_{2}\right)=\sum_{q, \pm} \gamma_{q} N_{q}^{ \pm} e^{ \pm i \omega_{q}\left(t_{1}-t_{2}\right)} G^{<}\left(t_{1}, t_{2}\right) \gamma_{q} .
$$

Within the SCBA scheme, the EOM of RSDM and expression of auxiliary density matrix are same as Eqs. (15) and (16), respectively. The difference is that self-energy to electron caused by EPI is evaluated within SCBA scheme. Since EPI does not cause any particle dissipation, $i \operatorname{Tr}\left[\varphi_{e p}(t)-\varphi_{e p}^{\dagger}(t)\right]$ should be zero in order to ensure the current continuity. Within the SCBA,

$$
\begin{aligned}
\operatorname{Tr}\left[\varphi_{e p}(t)\right]= & \operatorname{Tr}\left\{i \int _ { - \infty } ^ { t } d \tau \sum _ { q , \pm } N _ { q } ^ { \pm } \left[e^{\mp i \omega_{q}(\tau-t)} G^{<}(t, \tau)\right.\right. \\
& \left.\left.\times \gamma_{q} G^{>}(\tau, t)-e^{ \pm i \omega_{q}(\tau-t)} G^{>}(t, \tau) \gamma_{q} G^{<}(\tau, t)\right] \gamma_{q}\right\} \\
= & \operatorname{Tr}\left\{-i \int_{-\infty}^{t} d \tau \sum_{q, \pm} \gamma_{q} N_{q}^{ \pm}\left[e^{ \pm i \omega_{q}(\tau-t)} G^{>}(t, \tau)\right.\right. \\
& \left.\left.\times \gamma_{q} G^{<}(\tau, t)-e^{\mp i \omega_{q}(\tau-t)} G^{<}(t, \tau) \gamma_{q} G^{>}(\tau, t)\right]\right\} \\
= & \operatorname{Tr}\left[\varphi_{e p}^{\dagger}(t)\right],
\end{aligned}
$$

i.e., current continuity is satisfied within the SCBA scheme. The second equality in Eq. (23) is obtained by using the invariant property of trace under cyclic permutation; The third equality is ensured by the fact that the second line is the Hermitian of the first line. Unlike the Büttiker's virtual probe model which adopted virtual probes to mimic the dephasing process and the current continuity is satisfied by adjusting the chemical potential of each virtual probe, ${ }^{40}$ SCBA ensures the current continuity from a more natural way.

\section{NEGF-HEOM-WBL with EPI within scheme of LOE}

Even within the scheme of SCBA, evaluation of auxiliary density matrix $\varphi_{\alpha}(t)$ is nontrivial as the self-energy to electron caused by EPI is constructed by the dressed electron Green's function and time derivative of Green's function in return depends on the integral of self-energy $\Sigma_{\alpha}$ and Green's function $G$. This results in an infinite set of hierarchical equations. Fundamental reason of the infinite set of 
hierarchical equations is that, for the many-body problem, EOM of single-particle Green's function depends on twoparticle Green's function, EOM of two-particle Green's function relies on three-particle Green's functions and so on. Since it is nontrivial to solve the infinite set of hierarchical equations, an approximation is necessary for the practical studies. In this work, EPI is taken into account at the level of LOE. LOE expands the EPI dressed Green's function to the lowest order with respect to $\gamma_{q}$. The approximation is valid for systems with weak EPI. There are many types of experiments focus on single-molecule device connected to metallic contacts, which typically have weak EPI strength. Based on LOE, a time-dependent theory for quantum transport with weak EPI strength is established. The bare electron Green's function $G_{0}(t, \tau)$ and bare phonon Green's function $d_{0}(t, \tau)$ are used to construct the interacting self-energy $\Sigma_{e p}(t, \tau)$. Starting with $G_{0}(t, \tau)$ and within the scheme of LOE, the EPI dressed retarded electron Green's function is

$$
G^{r}(t, \tau)=G_{0}^{r}(t, \tau)+\left[G_{0}^{r} \Sigma_{e p}^{r} G_{0}^{r}\right](t, \tau),
$$

where the interacting self-energy is

$$
\Sigma_{e p}^{<}\left(t_{1}, t_{2}\right)=\sum_{q, \pm} \gamma_{q} N_{q}^{ \pm} e^{ \pm i \omega_{q}\left(t_{1}-t_{2}\right)} G_{0}^{<}\left(t_{1}, t_{2}\right) \gamma_{q} .
$$

Within the scheme of LOE, the EOM of RSDM is also same as Eq. (15), while the auxiliary density matrix $\varphi_{\alpha}(t)$ becomes

$$
\varphi_{\alpha}(t)=i \int_{-\infty}^{t} d \tau\left[G^{<}(t, \tau) \Sigma_{\alpha}^{>}(\tau, t)-G^{>}(t, \tau) \Sigma_{\alpha}^{<}(\tau, t)\right],
$$

for $\alpha=L, R$ and

$$
\varphi_{\alpha}(t)=i \int_{-\infty}^{t} d \tau\left[G_{0}^{<}(t, \tau) \Sigma_{\alpha}^{>}(\tau, t)-G_{0}^{>}(t, \tau) \Sigma_{\alpha}^{<}(\tau, t)\right]
$$

for $\alpha=e p$. All these auxiliary density matrices $\varphi_{\alpha}(t)$ are in order of $\gamma_{q}^{2}$ due to LOE. The auxiliary density matrix $\varphi_{L / R}(t)$ is responsible for the electron exchange between device and leads. Hence, the transient current going through each lead can be obtained by tracing auxiliary density matrix as described by Eq. (5). $\varphi_{e p}(t)$ represents the electron-phonon interaction which cause the inelastic scattering of electron and energy dissipation to phonon. Following the same procedure as Eq. (23), it can be proven that current continuity is also satisfied within the LOE scheme.

Since the phonon is treated as unperturbed by electron, this method cannot be applied to heating of the phonon subsystem. The energy transfers from electron is interpreted as being absorbed by the external thermal bath instantaneously, this can be regarded as the extremely damping limit. If heating of phonon system is considered, the number of phonon $N_{q}$ has to be time dependent. One way to include non-equilibrium heating is to give a rate equation for $N_{q}$, including an external damping rate of phonons. ${ }^{16,17,41}$

When $\alpha=L / R$, the auxiliary density matrix is related to the current flow. As described in Sec. II A, the lesser and greater self-energy are decomposed into two parts under the WBL approximation and Padé expansion for Fermi distribution function. The auxiliary density matrix can be rewritten as $\varphi_{\alpha}(t)=i[\sigma(t)-1 / 2] \Lambda_{\alpha}+\sum_{k} \varphi_{\alpha k}(t)$, where $\varphi_{\alpha k}(t)$ is the component of the first-tier auxiliary density matrix:

$$
\varphi_{\alpha k}(t)=-i \int_{-\infty}^{\infty} d \tau G^{r}(t, \tau) \Sigma_{\alpha k}^{+}(\tau, t) .
$$

Its expression is same as non-interacting case, i.e., Eq. (10), the difference is that the retarded Green's function is replaced by EPI dressed Green's function. EOM of the $\varphi_{\alpha k}(t)$ is

$$
\begin{aligned}
i \dot{\varphi}_{\alpha k}(t)= & -i \frac{2}{\beta} \eta_{k} \Lambda_{\alpha}-\left[G^{r}\left(\epsilon_{\alpha k}^{+}\right)\right]^{-1} \varphi_{\alpha k}(t) \\
& -i \int_{-\infty}^{\infty} d \tau \int_{-\infty}^{\infty} d t_{1} \Sigma_{e p}^{r}\left(t, t_{1}\right) G_{0}^{r}\left(t_{1}, \tau\right) \Sigma_{\alpha k}^{+}(\tau, t) .
\end{aligned}
$$

Compared with Eq. (11), an extra term arises in the presence of EPI, which is responsible for the dissipative effect of the electrons caused by EPI. Since the interacting self-energy is obtained by summation over the phonon modes, the last term in the RHS of above equation can be decomposed as $\sum_{q, x} \gamma_{q} \varphi_{\alpha k, q}^{x}(t)$, here $x= \pm$ and $\varphi_{\alpha k, q}^{x}(t)$ is

$$
\begin{aligned}
\varphi_{\alpha k, q}^{x}(t)= & i \int_{-\infty}^{t} d t_{1} \int_{-\infty}^{\infty} d \tau e^{i x \omega_{q}\left(t-t_{1}\right)}\left[N_{q}^{x} G_{0}^{<}\left(t, t_{1}\right)\right. \\
& \left.-N_{q}^{-x} G_{0}^{>}\left(t, t_{1}\right)\right] \gamma_{q} G_{0}^{r}\left(t_{1}, \tau\right) \Sigma_{\alpha k}^{+}(\tau, t),
\end{aligned}
$$

where $\varphi_{\alpha k, q}^{x}(t)$ is the second-tier auxiliary density matrix which contribute to the EOM of first-tier auxiliary density matrix. Similarly, taking time derivative of this term, the EOM of second-tier auxiliary density matrix is obtained

$$
\begin{aligned}
i \dot{\varphi}_{\alpha k, q}^{x}(t)= & {\left[N_{q}^{-x}+x \sigma_{0}(t)\right] \gamma_{q} \tilde{\varphi}_{\alpha k}(t) } \\
& -\left[G^{r}\left(\epsilon_{\alpha k}^{+}+x \omega_{q}\right)\right]^{-1} \varphi_{\alpha k, q}^{x}(t) \\
& -x \sum_{\alpha^{\prime} k^{\prime}} \varphi_{\alpha^{\prime} k^{\prime}, \alpha k}^{q x}(t),
\end{aligned}
$$

where $\varphi_{\alpha^{\prime} k^{\prime}, \alpha k}^{q x}(t)$ is the third-tier auxiliary density matrix. Use notation $\mathbf{a}=(\alpha k), \varphi_{\alpha^{\prime} k^{\prime}, \alpha k}^{q x}(t)$ is expressed as

$$
\begin{aligned}
\varphi_{\mathbf{a}^{\prime}, \mathbf{a}}^{q x}(t)= & i \int_{-\infty}^{t} d t_{1} e^{i x \omega_{q}\left(t-t_{1}\right)} \int_{-\infty}^{\infty} d t_{2} \Sigma_{\mathbf{a}^{\prime}}^{-}\left(t, t_{2}\right) \\
& \times G_{0}^{a}\left(t_{2}, t_{1}\right) \gamma_{q} \int_{-\infty}^{\infty} d \tau G_{0}^{r}\left(t_{1}, \tau\right) \Sigma_{\mathbf{a}}^{+}(\tau, t) .
\end{aligned}
$$

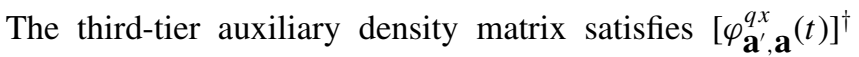
$=-\varphi_{\mathbf{a}, \mathbf{a}^{\prime}}^{q,-x}(t)$ and its EOM terminates to itself:

$$
i \dot{\varphi}_{\mathbf{a}^{\prime}, \mathbf{a}^{q x}}(t)=-\tilde{\varphi}_{\mathbf{a}^{\prime}}^{\dagger}(t) \gamma_{q} \tilde{\varphi} \mathbf{a}^{\prime}(t)+\left[\epsilon_{\mathbf{a}^{\prime}}^{-}-x \omega_{q}-\epsilon_{\mathbf{a}}^{+}\right] \varphi_{\mathbf{a}^{\prime}, \mathbf{a}^{q x}}^{(t),}
$$

where $\tilde{\varphi}_{\alpha^{\prime} k^{\prime}}(t)$ is the non-interacting first-tier auxiliary density matrix as described in Sec. II A.

$\varphi_{e p}(t)$ is the dissipation term arises from EPI, it is related to the energy exchange between electron and phonon, i.e., phonon absorption and emission. As the interacting self-energy is decomposed according to phonon mode, this EPI auxiliary density matrix can be decomposed as, $\varphi_{e p}(t)$ $=\sum_{q x} \varphi_{q}^{x}(t) \gamma_{q}$, where $x= \pm$ and

$$
\begin{aligned}
\varphi_{q}^{x}(t)= & i \int_{-\infty}^{t} d \tau e^{i x \omega_{q}(t-\tau)}\left[N_{q}^{x} G_{0}^{<}(t, \tau) \gamma_{q} G_{0}^{>}(\tau, t)\right. \\
& \left.-N_{q}^{-x} G_{0}^{>}(t, \tau) \gamma_{q} G_{0}^{<}(\tau, t)\right] .
\end{aligned}
$$


It is obvious that $\varphi_{q}^{x}(t)$ satisfies the Hermitian relationship $\left[\varphi_{q}^{x}(t)\right]^{\dagger}=\varphi_{q}^{-x}(t)$. Therefore $\quad\left[\varphi_{q}(t)\right]^{\dagger}=\left[\varphi_{q}^{x}(t)+\varphi_{q}^{-x}(t)\right]^{\dagger}$ $=\varphi_{q}(t)$. This actually ensures the current continuity. Now, the main task is to obtain a solution to $\varphi_{q}^{x}(t)$. The EOM of $\varphi_{q}^{x}(t)$ is expressed as

$$
\begin{aligned}
i \dot{\varphi}_{q}^{x}(t)= & -\left[N_{q}^{x} \sigma_{0}(t) \gamma_{q} \bar{\sigma}_{0}(t)-N_{q}^{-x} \bar{\sigma}_{0}(t) \gamma_{q} \sigma_{0}(t)\right] \\
& -\left[x \omega_{q}-\mathscr{L}\right] \varphi_{q}^{x}(t)+\sum_{\alpha k}\left\{\left[\varphi_{\alpha k, q}^{-x}(t)\right]^{\dagger}-\varphi_{\alpha k, q}^{x}(t)\right\},
\end{aligned}
$$

where $\bar{\sigma}_{0}=1-\sigma_{0}$ and $\sigma_{0}=-i G_{0}^{<}(t, t)$ is the noninteracting RSDM. The Liouville operator $\mathscr{L}$ is defined as $\mathscr{L} A=[h(0)-i \Lambda] A-A[h(0)+i \Lambda]$.

Hence, NEGF-HEOM-WBL scheme terminates at the third-tier when EPI is taken into consideration. In practice, the EOMs of RSDM and all the auxiliary density matrices are propagated together. Time-dependent charge density and current are then evaluated via the time-dependent RSDM and auxiliary density matrices. The most time-consuming and memory requirement part is the evaluation of $\varphi_{\mathbf{a}^{\prime}}^{q x}(t)$. According to the Hermitian relation between $\varphi_{\mathbf{a}^{\prime}, \mathbf{a}^{q x}}^{(t)}$ and $\varphi_{\mathbf{a}^{\prime}, \mathbf{a}}^{q,-x}(t)$, only $\varphi_{\mathbf{a}^{\prime}, \mathbf{a}^{q+}}^{q+}(t)$ has to be propagated. Consequently, a total of $4 N_{k}^{2} N_{q}$ third-tier auxiliary density matrices have to be evaluated, where $N_{k}$ and $N_{q}$ are number of terms in Padé expansion and number of phonon modes, respectively.

It should be pointed out that the EOM of density matrix can also be derived from the standard Kadanoff-Baym (KB) kinetic equations ${ }^{39}$ for lesser Green's function since $\sigma(t)$ $=-\left.i G^{<}\left(t, t^{\prime}\right)\right|_{t^{\prime}=t}$. The KB kinetic equations are

$$
\begin{aligned}
i \partial_{t} G^{<}\left(t, t^{\prime}\right) & =h G^{<}\left(t, t^{\prime}\right)+I_{1}^{<}\left(t, t^{\prime}\right), \\
-i \partial_{t^{\prime}} G^{<}\left(t, t^{\prime}\right) & =G^{<}\left(t, t^{\prime}\right) h\left(t^{\prime}\right)+I_{2}^{<}\left(t, t^{\prime}\right),
\end{aligned}
$$

where $I_{1}^{<}\left(t, t^{\prime}\right)$ and $I_{2}^{<}\left(t, t^{\prime}\right)$ are the so-called quantum collision terms which have the forms

$$
\begin{aligned}
I_{1}^{<}\left(t, t^{\prime}\right)= & \int d t_{1} \Sigma^{r}\left(t, t_{1}\right) G^{<}\left(t_{1}, t^{\prime}\right) \\
& +\int d t_{1} \Sigma^{<}\left(t, t_{1}\right) G^{a}\left(t_{1}, t^{\prime}\right)
\end{aligned}
$$

and $I_{2}^{<}\left(t, t^{\prime}\right)=\left[I_{1}^{<}\left(t^{\prime}, t\right)\right]^{\dagger}$. Compared with the KB kinetic equations, the NEGF-HEOM-WBL method has the advantage that all the variables, density matrix, and auxiliary ones, in the NEGF-HEOM-WBL are one-time functions, and time complexity of propagation of these variables is linear-scaling against the simulation time. While the variables involved in the KB kinetic equations, such lesser Green's functions and quantum collision terms, are two-time functions. Numerical implementation of KB kinetic equations requires calculation and storage of Green's function $G^{<}\left(t, t^{\prime}\right)$ for different $t$ and $t^{\prime}$, making the time and memory costs goes up quickly with larger $t$ and $t^{\prime}$. Moreover, the simulation time of integration in quantum collision terms increases with $t$ and $t^{\prime}$. Therefore, the time-complexity and memory costs of KB kinetic equations is superlinear against the simulation time. ${ }^{42}$ Hence, the NEGFHEOM-WBL scheme developed in this work is more efficient than the standard KB kinetic equations.

\section{Initial condition}

To ensure the accuracy of the time propagation, initial value should be well prepared. At the initial time, the system is at equilibrium state and each physical quantity stays unchange with time. Thus, the time-derivative of the density matrix and auxiliary density matrix should be zero. Hence, initial condition for density matrix and auxiliary density matrix are obtained by solving their linear or nonlinear equations. According to their EOMs, it is straightforward to show that $\varphi_{\alpha k, \alpha^{\prime} k^{\prime}}^{q x}(0)$ is

$$
\varphi_{\alpha^{\prime} k^{\prime}, \alpha k}^{q x}(0)=\frac{1}{\epsilon_{\alpha^{\prime} k^{\prime}}^{-}-x \omega_{q}-\epsilon_{\alpha k}^{+}}\left[\tilde{\varphi}_{\alpha^{\prime} k^{\prime}}(0)\right]^{\dagger} \gamma_{q} \tilde{\varphi}_{\alpha k}(0),
$$

where $\tilde{\varphi}_{\alpha k}(0)$ is $-i \frac{2}{\beta} \eta_{k} G^{r}\left(\epsilon_{\alpha k}\right) \tilde{\Lambda}_{\alpha}$. Substitute Eq. (36) to Eq. (29), $\varphi_{\alpha k}^{x}\left(\omega_{q}, 0\right)$ can be expressed as

$$
\begin{aligned}
\varphi_{\alpha k, q}^{x}(0)= & G^{r}\left(\epsilon_{\alpha k}^{+}+x \omega_{q}\right)\left\{\left[N_{q}^{-x}+x \sigma_{0}\right]\right. \\
& \left.-x \sum_{\alpha^{\prime} k^{\prime}} \frac{1}{\epsilon_{\alpha^{\prime} k^{\prime}}^{-}-x \omega_{q}-\epsilon_{\alpha k}^{+}}\left[\tilde{\varphi}_{\alpha^{\prime} k^{\prime}}(0)\right]^{\dagger}\right\} \gamma_{q} \tilde{\varphi}_{\alpha k}(0) .
\end{aligned}
$$

According to the EOM of the first-tier auxiliary density matrix $\varphi_{\alpha k}(t)$, the initial condition for first-tier auxiliary density matrix $\varphi_{\alpha k}(0)$ can be obtained after a simple algebra,

$$
\varphi_{\alpha k}(0)=-i \frac{2}{\beta} \eta_{k} \tilde{G}^{r}\left(\epsilon_{\alpha k}\right) \tilde{\Lambda}_{\alpha},
$$

where $\tilde{G}^{r}\left(\epsilon_{\alpha k}\right)=G^{r}\left(\epsilon_{\alpha k}\right)\left[1+\Sigma^{r}\left(\epsilon_{\alpha k}\right) G^{r}\left(\epsilon_{\alpha k}\right)\right] . \quad \Sigma^{r}\left(\epsilon_{\alpha k}\right)$ is the retarded electron-phonon interacting self-energy, which accounts for the dissipative effect due to EPI. Its expression is

$$
\begin{aligned}
\Sigma^{r}\left(\epsilon_{\alpha k}\right)= & \sum_{q x} \gamma_{q} G^{r}\left(\epsilon_{\alpha k}^{+}+x \omega_{q}\right)\left\{\left[N_{q}^{-x}+x \sigma_{0}\right]\right. \\
& \left.-\sum_{\alpha^{\prime} k^{\prime}} \frac{x}{\epsilon_{\alpha^{\prime} k^{\prime}}^{-}-x \omega_{q}-\epsilon_{\alpha k}^{+}}\left[\tilde{\varphi}_{\alpha^{\prime} k^{\prime}}(0)\right]^{\dagger}\right\} \gamma_{q} .
\end{aligned}
$$

Finally, initial value of RSDM is evaluated from this dressed Green's function

$$
\sigma(0)=\frac{1}{2} \mathbf{I}+\sum_{\alpha k} \frac{2}{\beta} \eta_{k} \operatorname{Re}\left[\tilde{G}^{r}\left(\epsilon_{\alpha k}\right)\right],
$$

where $\mathbf{I}$ is identity matrix.

For the initial value of EPI functional $\varphi_{q}^{x}(0)$, it satisfied the nonlinear equation

$$
\begin{aligned}
{\left[x \omega_{q}-\mathscr{L}\right] \varphi_{q}^{x}(0)=} & {\left[N_{q}^{-x} \bar{\sigma}_{0}(0) \gamma_{q} \sigma_{0}(0)-N_{q}^{x} \sigma_{0}(t) \gamma_{q} \bar{\sigma}_{0}(0)\right] } \\
& +\sum_{\alpha k}\left\{\left[\varphi_{\alpha k, q}^{-x}(0)\right]^{\dagger}-\varphi_{\alpha k, q}^{x}(0)\right\}
\end{aligned}
$$

This nonlinear equation can be solved by the bi-conjugate gradient stability method. ${ }^{43}$ Using the method described above, the initial condition for density matrices and auxiliary density matrices can be readily determined. 


\section{NUMERICAL APPLICATION}

To show the validity and gain further insight of proposed method, two simple models are used to illustrate the physics of proposed theory. In the following model simulation, linear drop of voltage across the device region is assumed.

\section{A. One-level model}

The NEGF-HEOM-WBL scheme is applied to one-level model which is coupled to one phonon mode, i.e.,

$$
H_{D}=\epsilon_{0} d^{\dagger} d+\omega b^{\dagger} b+\gamma d^{\dagger} d\left(b^{\dagger}+b\right) .
$$

In the simulation, on-site energy $\epsilon_{0}=0 \mathrm{eV}$; line-width function is $\Lambda_{L}=\Lambda_{R}=0.1 \mathrm{eV}$; Time-dependent bias voltage $V(t)=0.1\left(1-e^{-t / 0.01}\right) \mathrm{eV}$ is applied on the system; Temperature $T$ is set to $300 \mathrm{~K}$.

First, steady state current is calculated from NEGFHEOM-WBL and compared with results obtained from MeirWingreen formulation. ${ }^{1,39}$ Table I shows the comparison between the two methods. $I_{A}$ is evaluated from Meir-Wingreen formula; $I_{B}$ is obtained by NEGF-HEOM-WBL. A small term in retarded self-energy $\Sigma_{e p}^{r}(\epsilon)$ which is proportional to the coupling constant and phonon frequency in the MeirWingreen calculation is neglected for simplification ${ }^{38}$ (See the Appendix for details). This is the reason why there is a slight difference between the currents calculated from the two method and the steady state current from Meir-Wingreen formula approaches the NEGF-HEOM-WBL result when $\gamma^{2} \omega$ is very small. The comparison in Table I indicates the validity of the steady state current obtained from NEGF-HEOM-WBL approach. Next, transient current is demonstrated, Fig. 3 shows the transient currents obtained from NEGF-HEOMWBL corresponding to the cases listed in Table I. With the same phonon frequency, the system with larger EPI coupling constant results in lower current. Besides, when the EPI coupling constants are same, the system with lower phonon frequency has smaller current. The reason is that the occupation number is larger for the system with lower frequency which results in larger scattering effect to electron.

Figure 4 shows the temperature effects on current. Since the occupation number of phonon varies at different temperature, in turn affects the EPI self-energy, i.e., changes the dissipative effects on electron and affects the transport properties.

TABLE I. Steady state current calculated from two methods on different phonon frequencies and electron-phonon coupling constant. $I_{A}$ is evaluated from Meir-Wingreen formula; $I_{B}$ is obtained by the NEGF-HEOM-WBL. The results indicate the validity of the steady state current obtained from the NEGF-HEOM-WBL method. See text for other parameters.

\begin{tabular}{lcrrrr}
\hline \hline$\omega(\mathrm{eV})$ & $\gamma(\mathrm{eV})$ & $\gamma^{2} \omega$ & $I_{A}(\mathrm{nA})$ & $I_{B}(\mathrm{nA})$ & $\Delta(\%)^{\mathrm{a}}$ \\
\hline 0.10 & 0.10 & $1 \times 10^{-3}$ & 3493.67 & 3373.92 & 3.43 \\
0.05 & 0.10 & $5 \times 10^{-4}$ & 3052.55 & 2967.69 & 2.78 \\
0.05 & 0.05 & $1.25 \times 10^{-4}$ & 3498.08 & 3467.78 & 0.87 \\
0.005 & 0.05 & $1.25 \times 10^{-5}$ & 1859.55 & 1857.12 & 0.13 \\
0.01 & 0.01 & $1 \times 10^{-6}$ & 3599.30 & 3598.99 & 0.01 \\
0.0 & 0.0 & 0 & 3634.61 & 3634.48 & $\ldots$ \\
\hline \hline
\end{tabular}

${ }^{\mathrm{a}} \Delta=\left|I_{A}-I_{B}\right| / I_{A} \times 100 \%$.

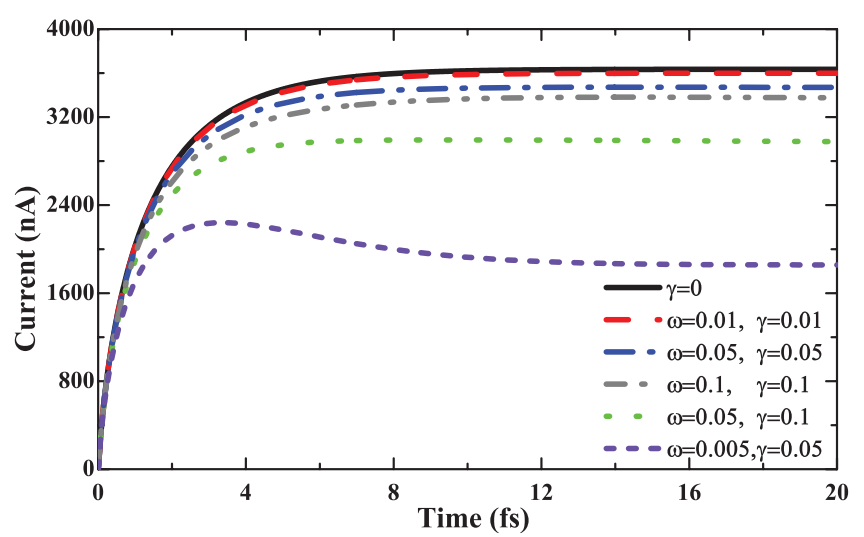

FIG. 3. Transient current corresponding to the cases listed in Table I.

Our results show that conductance decreases with increasing temperature. This phenomenon arises from the fact that higher temperature excites more phonons, i.e., increases $N_{q}$, resulting in stronger scattering effect to electron.

\section{B. Multi-sites model: Gold wire}

One-dimensional gold wire is chosen as a simple illustration of our method. In tight-binding model, electronic Hamiltonian of gold wire in device region is

$$
H_{D}=\sum_{i} \epsilon_{i} d_{i}^{\dagger} d_{i}+\sum_{i j} t_{i j}\left(d_{i}^{\dagger} d_{j}+\text { h.c. }\right)
$$

where $\epsilon_{i}$ is the on-site energy and $t_{i j}$ is the hopping element between localized orbitals $|i\rangle$ and $|j\rangle$. The hopping element explicitly depends on the displacement of nuclear (phonon). SuSchrieffer-Heeger ( $\mathrm{SSH}$ ) model is chosen to describe the displacement effect on electron hopping. ${ }^{44} \mathrm{SSH}$ model expands the hopping element to first order in terms of displacement, $t_{i j}=t_{i, j}^{0}+t_{i j}^{\prime}\left(Q_{i}-Q_{i+1}\right)$ where $t_{i j}^{\prime}=-t_{j i}^{\prime}$. And nuclear is described by the following Hamiltonian:

$$
H_{p h}=\sum_{i} \frac{1}{2} m_{i} \dot{Q}_{i}^{2}+\frac{1}{2} k_{i, i+1}\left(Q_{i}-Q_{i+1}\right)^{2},
$$

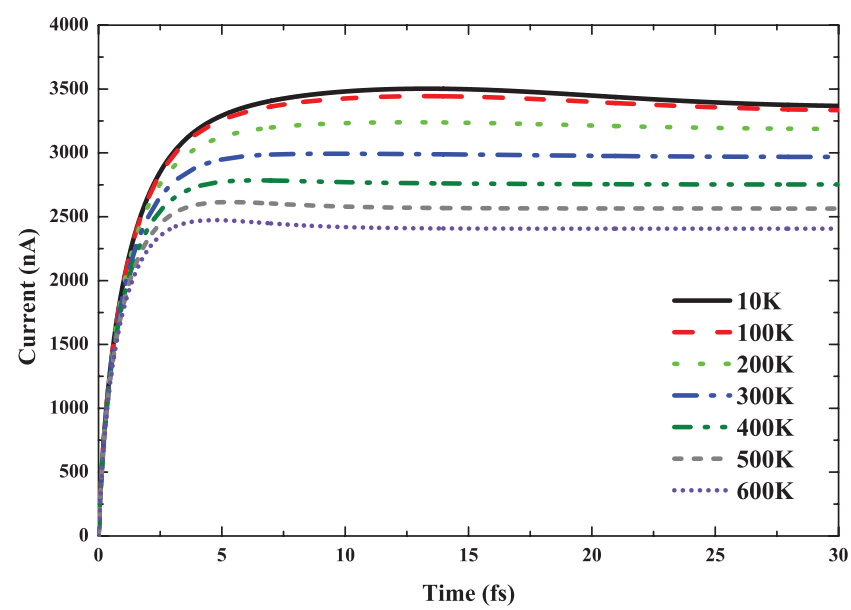

FIG. 4. Transient current at different temperature. On-site energy is 0 ; $\Lambda_{L}=\Lambda_{R}=0.1 ; \omega=0.05$ and $\gamma=0.1$. Voltage is $V(t)=0.1\left(1-e^{-t / 0.01}\right)$. 


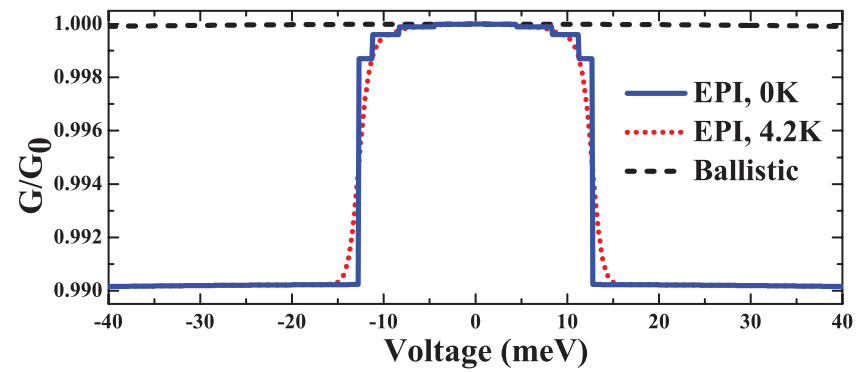

FIG. 5. Differential conductance for a 10 -atom wire, $G_{0}$ is the quantum conductance $2 e^{2} / h$.

where $m_{i}$ is the mass of $\mathrm{i} t h$ nuclear and $k_{i, i+1}$ is the spring constant between two nuclear and only nearest neighbor spring is included in this model. In the second quantization expression, the hopping element is $t_{i, j}=t_{i, j}^{0}+\sum_{q} \gamma_{i j}^{q}$ $\left(b_{q}^{\dagger}+b_{q}\right)$. The EPI coupling matrix $\gamma_{i j}^{q}$ is defined as $\gamma_{i j}^{q}$ $=t_{i j}^{\prime} \frac{\sqrt{m_{j}} e_{i q}-\sqrt{m_{i}} e_{j q}}{\sqrt{m_{i} m_{j}}} \sqrt{\frac{\hbar}{2 \omega_{q}}}$, where $e_{i q}$ is the ith element of normal mode vector $\mathbf{e}_{q}$. The normal modes and phonon frequency can be obtained by diagonalizing Hessian matrix which is related to the spring constant and nuclear mass.

Parameters used in this simulation are as follows: $\epsilon_{i}$ $=0 \mathrm{eV} ; t^{0}=1.0 \mathrm{eV} ; t^{\prime}=0.6 \mathrm{eV} / \AA ; \quad m=197 \mathrm{amu}$ and $k=2.0 \mathrm{eV} / \AA$, which is same as Ref. 17. In the following simulation, the atoms at two ends of the device region are fixed. Thus, only $N-2$ atoms are allowed to vibrate, where $N$ is the number of atoms in the device. Figure 5 plots the conductance of a 10-atom gold wire, A notable difference can be found between zero temperature and finite temperature conductance. The steps of conductance at zero temperature is smoothed at finite temperature. At zero temperature, four conductance steps are found due to the excitation of four phonon modes at $4.44 \mathrm{meV}, 8.34 \mathrm{meV}, 11.24 \mathrm{meV}$ and $12.79 \mathrm{meV}$, respectively. When bias voltage $V$ is larger than phonon energy $\omega_{q}$, transporting electron can excite the corresponding phonon mode and provide an inelastic transport path, which is represented by a step in differential conductance at zero temperature. ${ }^{45}$ Compared to ballistic transport, it is indicated that these four phonon modes have major contribution to the conductance changes. The conductance calculation agrees qualitatively with experiment. ${ }^{15}$

Figure 6 shows the transient current of various number of atoms at bias voltage $15 \mathrm{meV}$ at which all the phonon modes are excited. Since we assume phonon system is in equilibrium, the transient regime for electron transport is in the time scale of femtosecond. The inset plots steady state current against number of gold atom included in device region. As shown from the inset of Fig. 6, the current and hence conductance drops linearly with the increasing number of atoms. This indicates that EPI has a significant effect on electron transport even though the EPI coupling constant is weak. In this study, heating effect is not included, the phonon occupation number is constant along the time evolution. However, if heating of phonon system is included, the phonon occupation number becomes bias and time dependent, which will also affects the transient and steady state current.

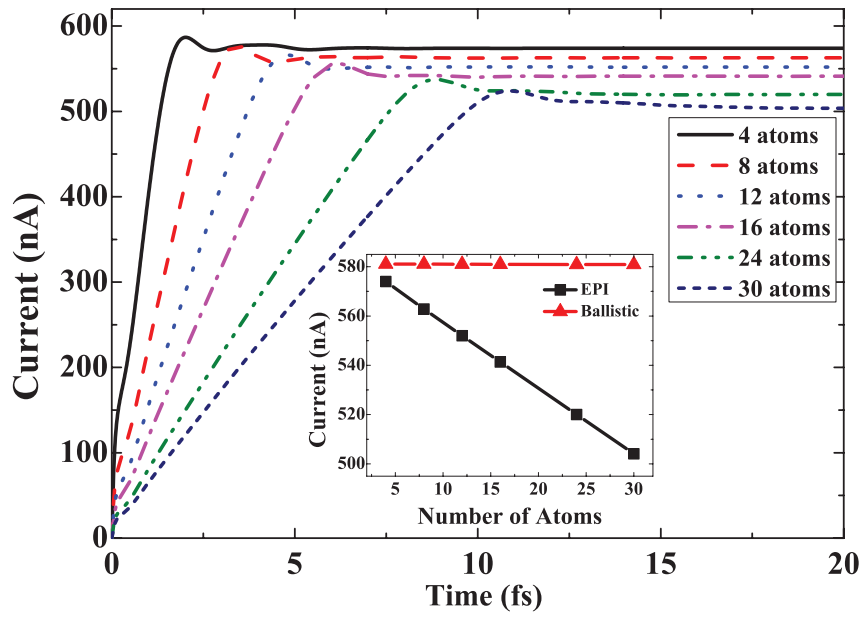

FIG. 6. Transient current of various number of atoms within SSH model; The inset graph is the corresponding steady state current against number of atoms; bias voltage is $15 \mathrm{meV}$.

\section{SUMMARY}

In summary, a time-dependent transport theory is established to investigate the dissipative transport in the presence of EPI. The EPI Green's function is perturbatively evaluated by the LOE with respect to EPI coupling constant $\gamma^{q}$. This LOE approximation ensures the current continuity law and is valid for the weak coupling system. Starting from LOE and single-electron Hamiltonian, EOMs of density matrix and corresponding auxiliary density matrices are formulated, resulting in a three-tier NEGF-HEOM-WBL scheme. Numerical studies indicated inelastic effect have significant effect on current compared to ballistic transport.

Although the results shown in this work are only for model systems, the methodology can be readily extended to TDDFT due to the fact that TDDFT can be regarded as an effective single-electron model. Within TDDFT, Kohn-Sham Hamiltonian reads $H_{e}^{K S}=\sum_{i j} h_{i j} d_{i}^{\dagger} d_{j}$, where $h$ is KohnSham Fock matrix. $h_{i j}=\langle i|\hat{h}(\mathbf{r})| j\rangle$ is the element of effective single-electron Fock operator $\hat{h}(\mathbf{r})$ with respect to atomic basis. $\hat{h}(\mathbf{r})=-\frac{1}{2} \nabla^{2}+V_{e f f}(\mathbf{r})$, here $V_{e f f}(\mathbf{r})$ is the effective potential which contains external potential $V_{\text {ext }}(\mathbf{r})$, Hartree potential $V_{H}(\mathbf{r})$, and exchange-correlation potential $V_{X C}(\mathbf{r})$. Since atomic basis set and Fock operator depend on atomic nuclei, phonon Hamiltonian and electron-phonon interaction Hamiltonian arise naturally from Born-Oppenheimer approximation. Starting from total energy surface $E(\mathbf{R})$, phonon modes are calculated from the mass-weighted second derivative of $E(\mathbf{R})$, i.e., Hessian matrix. And electron-phonon coupling matrix is obtained by expanding electronic Kohn-Sham Hamiltonian respect to nuclei oscillation. ${ }^{28}$ With the KohnSham Fock matrix, phonon frequencies and electron-phonon coupling matrix known, the NEGF-HEOM-WBL method developed in Sec. II, can be readily applied for the investigation of dissipative time-dependent quantum transport through realistic systems from first-principles.

In this work, the phonon is assumed to be in equilibrium. This method can be extended to study the time-dependent heating effect when current flows through the junction. The 
simplest way to include non-equilibrium phonon is to introduce a rate equation for phonon occupation number $N_{q}$, including a external damping rate. When the rate equation is included, $N_{q}$ becomes bias and time dependent, it can be evaluated by the propagation method same as the density matrix. Furthermore, the time derivative of third-tier auxiliary density matrix is small and can be safely neglected, the third-tier auxiliary density matrix can then be expressed by non-interacting auxiliary density matrix as shown by Eq. (36). This simplification can greatly reduce the computational time and memory cost and can be applied on larger realistic systems combined with first-principles method. Besides, similar statistics of photons and phonons implies that the developed framework can also be readily extended to treat the photon excitation and emission in molecular junction.

\section{ACKNOWLEDGMENTS}

Support from the Hong Kong Research Grant Council (Contract Nos. HKU7009/09P, 7009/12P, 7007/11P, and HKUST9/CRF/11G), and the University Grant Council (Contract No. AoE/P-04/08) is gratefully acknowledged.

\section{APPENDIX: STEADY STATE CURRENT CALCULATION FROM MEIR-WINGREEN FORMULA WITH EPI}

Within Meir-Wingreen formula, the steady state current $I_{\alpha}$ can be expressed as ${ }^{1,39}$

$$
I_{\alpha}=\int d \epsilon T_{\alpha}(\epsilon)
$$

where $T_{\alpha}(\epsilon)=\operatorname{Tr}\left[\Sigma_{\alpha}^{<}(\epsilon) G^{>}(\epsilon)-\Sigma_{\alpha}^{>}(\epsilon) G^{<}(\epsilon)\right]$. The lesser and greater Green's functions are related to the retarded and advanced ones via the equation

$$
G^{\gtrless}(\epsilon)=G^{r}(\epsilon)\left[\Sigma_{L}^{\gtrless}(\epsilon)+\Sigma_{R}^{\gtrless}(\epsilon)+\Sigma_{e p}^{\gtrless}(\epsilon)\right] G^{a}(\epsilon) .
$$

Substitute Eq. (A2) into Eq. (A1), the expression for current $I_{\alpha}$ can be divided into two parts. Taking $I_{L}$ for instance, the expression for current $I_{L}$ is then rewritten as $I_{L}=I_{L}^{e l}+I_{L}^{\text {inel }}$ where

$$
\begin{gathered}
I_{L}^{e l}=\int d \epsilon\left[f_{L}(\epsilon)-f_{R}(\epsilon)\right] \operatorname{Tr}\left[\Lambda_{L} G^{r}(\epsilon) \Lambda_{R} G^{a}(\epsilon)\right], \\
I_{L}^{\text {inel }}=\int d \epsilon \operatorname{Tr}\left[\Sigma_{L}^{<}(\epsilon) G^{r}(\epsilon) \Sigma_{e p}^{>}(\epsilon) G^{a}(\epsilon)\right. \\
\left.-\Sigma_{L}^{>}(\epsilon) G^{r}(\epsilon) \Sigma_{e p}^{<}(\epsilon) G^{a}(\epsilon)\right] .
\end{gathered}
$$

Here $I_{L}^{e l}$ is the elastic part, which is same as that in the Landauer-Büttiker formalism; $I_{L}^{\text {inel }}$ is the inelastic part of the current due to EPI.

Next, expression for current under LOE will be derived. With LOE, the retarded and lesser interacting self-energies are

$$
\Sigma_{e p}^{<}(\epsilon)=\sum_{q, \pm} \gamma_{q} N_{q}^{ \pm} G_{0}^{<}\left(\epsilon \pm \omega_{q}\right) \gamma_{q},
$$

$$
\begin{aligned}
\Sigma_{e p}^{r}(\epsilon)= & \sum_{q, \pm} \gamma_{q}\left[N_{q}^{\mp} G_{0}^{r}\left(\epsilon \pm \omega_{q}\right) \pm i \int d \epsilon^{\prime} G_{0}^{<}\left(\epsilon-\epsilon^{\prime}\right)\right. \\
& \left.\times \frac{1}{\epsilon^{\prime} \mp \omega_{q}+i \eta}\right] \gamma_{q}
\end{aligned}
$$

where $G_{0}^{r}(\epsilon)$ and $G_{0}^{<}(\epsilon)$ are the Green's functions without EPI,

$$
\begin{aligned}
G_{0}^{r}(\epsilon) & =\left[\epsilon \mathbf{I}-h_{D}-\Sigma_{L}^{r}(\epsilon)-\Sigma_{R}^{r}(\epsilon)\right]^{-1}, \\
G_{0}^{<}(\epsilon) & =G_{0}^{r}(\epsilon)\left[\Sigma_{L}^{<}(\epsilon)+\Sigma_{R}^{<}(\epsilon)\right] G_{0}^{a}(\epsilon) .
\end{aligned}
$$

With WBL approximation for leads, retarded and lesser selfenergies are $\Sigma_{\alpha}^{r}(\epsilon)=-i \Lambda_{\alpha}$ and $\Sigma_{\alpha}^{<}(\epsilon)=2 i f_{\alpha}(\epsilon) \Lambda_{\alpha}$, respectively.

By using the identity $\frac{1}{\epsilon+i \eta}=P \frac{1}{\epsilon}-i \pi \delta(\epsilon)$, where $P$ stands for Cauchy principal value integral, Eq. (A6) is transformed to

$$
\begin{aligned}
\Sigma_{e p}^{r}(\epsilon)= & \sum_{q, \pm} \gamma_{q}\left[N_{q}^{\mp} G_{0}^{r}\left(\epsilon \pm \omega_{q}\right) \pm G_{0}^{<}\left(\epsilon \mp \omega_{q}\right)\right] \gamma_{q} \\
& +\delta \Sigma_{e p}^{r}(\epsilon)
\end{aligned}
$$

where

$$
\delta \Sigma_{e p}^{r}(\epsilon) \equiv i \sum_{q, \pm}( \pm) P \int d \epsilon^{\prime} \gamma_{q} \frac{1}{\epsilon^{\prime}} G_{0}^{<}\left(\epsilon-\epsilon^{\prime} \mp \omega_{q}\right) \gamma_{q} .
$$

This term can be approximated as

$$
\delta \Sigma_{e p}^{r}(\epsilon) \simeq-2 i \sum_{q} P \int d \epsilon^{\prime} \frac{1}{\epsilon^{\prime}} \gamma_{q} \frac{\partial G_{0}^{<}\left(\epsilon-\epsilon^{\prime}\right)}{\partial \epsilon} \omega_{q} \gamma_{q}
$$

It is obvious that this term is proportional to the phonon frequencies and electron-phonon coupling constant $\gamma_{q}^{q} \omega_{q}$, which is small compared to the electron energy scale. Therefore, it is reasonable to omit this term from the retarded self-energy, which simplifies the calculation of retarded self-energy. ${ }^{38}$

Within LOE, the EPI dressed retarded Green's function is then

$$
G^{r}(\epsilon)=G_{0}^{r}(\epsilon)+G_{0}^{r}(\epsilon) \Sigma_{e p}^{r}(\epsilon) G_{0}^{r}(\epsilon) .
$$

Substitute Eq. (A11) into Eq. (A3), the elastic current is further split into two parts $I_{L}^{e l}=I_{L}^{e l, 0}+\delta I_{L}^{e l}$,

$$
\begin{aligned}
I_{L}^{e l, 0}= & \int d \epsilon\left[f_{L}(\epsilon)-f_{R}(\epsilon)\right] \operatorname{Tr}\left[\Lambda_{L} G_{0}^{r}(\epsilon) \Lambda_{R} G_{0}^{a}(\epsilon)\right], \\
\delta I_{L}^{e l}= & 2 \int d \epsilon\left[f_{L}(\epsilon)-f_{R}(\epsilon)\right] \\
& \times \operatorname{Tr}\left[\Lambda_{L} G_{0}^{r}(\epsilon) \Sigma_{e p}^{r}(\epsilon) G_{0}^{r}(\epsilon) \Lambda_{R} G_{0}^{a}(\epsilon)\right],
\end{aligned}
$$

The inelastic part of current within LOE is

$$
\begin{aligned}
I_{L}^{i n e l}= & \int d \epsilon \operatorname{Tr}\left[\Sigma_{L}^{<}(\epsilon) G_{0}^{r}(\epsilon) \Sigma_{e p}^{>}(\epsilon) G_{0}^{a}(\epsilon)\right. \\
& \left.-\Sigma_{L}^{>}(\epsilon) G_{0}^{r}(\epsilon) \Sigma_{e p}^{<}(\epsilon) G_{0}^{a}(\epsilon)\right] .
\end{aligned}
$$

Obviously, the elastic and inelastic parts of current are both in order of $\gamma_{q}^{2}$ due to LOE.

The numerical procedure for the calculation of current is as followed: (a) First, retarded and lesser Green's functions without EPI are obtained from Eq. (A7); (b) then, retarded and lesser self-energies due to EPI are evaluated through 
Eqs. (A5) and (A8), respectively; and (c) finally, current is calculated from Eqs. (A12) and (A13).

${ }^{1}$ A.-P. Jauho, N. S. Wingreen, and Y. Meir, Phys. Rev. B 50, 5528 (1994).

${ }^{2}$ S. Kurth, G. Stefanucci, C.-O. Almbladh, A. Rubio, and E. K. U. Gross, Phys. Rev. B 72, 035308 (2005).

${ }^{3}$ X. Zheng, F. Wang, C. Y. Yam, Y. Mo, and G. H. Chen, Phys. Rev. B 75, 195127 (2007)

${ }^{4}$ X. Zheng, G. H. Chen, Y. Mo, S. Koo, H. Tian, C. Y. Yam, and Y. Yan, J. Chem. Phys. 133, 114101 (2010).

${ }^{5}$ Y. Zhang, S. Chen, and G. H. Chen, Phys. Rev. B 87, 085110 (2013).

${ }^{6}$ H. Xie, F. Jiang, H. Tian, X. Zheng, Y. Kwok, S. Chen, C. Y. Yam, Y. Yan, and G. H. Chen, J. Chem. Phys. 137, 044113 (2012).

${ }^{7}$ D. G. Tempel, M. A. Watson, R. Olivares-Amaya, and A. Aspuru-Guzik, J. Chem. Phys. 134, 074116 (2011).

${ }^{8}$ M. D. Ventra and T. N. Todorov, J. Phys.: Condens. Matter 16, 8025 (2004).

${ }^{9}$ S. Chen, H. Xie, Y. Zhang, X. Cui, and G. H. Chen, Nanoscale 5, 169 (2013).

${ }^{10}$ X. Zheng, C. Y. Yam, F. Wang, and G. H. Chen, Phys. Chem. Chem. Phys. 13, 14358 (2011).

${ }^{11}$ E. Runge and E. K. U. Gross, Phys. Rev. Lett. 52, 997 (1984).

${ }^{12}$ C. Y. Yam, Y. Mo, F. Wang, X. Li, G. H. Chen, X. Zheng, Y. Matsuda, J. Tahir-Kheli, and W. A. Goddard III, Nanotechnology 19, 495203 (2008).

${ }^{13}$ J. Jin, X. Zheng, and Y. Yan, J. Chem. Phys. 128, 234703 (2008).

${ }^{14}$ M. Kumar, R. Avriller, A. L. Yeyati, and J. M. van Ruitenbeek, Phys. Rev. Lett. 108, 146602 (2012).

${ }^{15}$ N. Agraï, C. Untiedt, G. Rubio-Bollinger, and S. Vieira, Phys. Rev. Lett. 88, 216803 (2002)

${ }^{16}$ M. Paulsson, T. Frederiksen, and M. Brandbyge, J. Phys.: Conf. Ser. 35, 247 (2006).

${ }^{17}$ M. Paulsson, T. Frederiksen, and M. Brandbyge, Phys. Rev. B 72, 201101 (2005).

${ }^{18}$ M. Paulsson, T. Frederiksen, H. Ueba, N. Lorente, and M. Brandbyge, Phys. Rev. Lett. 100, 226604 (2008).

${ }^{19}$ J. K. Viljas, J. C. Cuevas, F. Pauly, and M. Häfner, Phys. Rev. B 72, 245415 (2005).

${ }^{20}$ M. Galperin, M. A. Ratner, and A. Nitzan, J. Phys.: Condens. Matter 19, 103201 (2007)
${ }^{21}$ H. Mera, M. Lannoo, C. Li, N. Cavassilas, and M. Bescond, Phys. Rev. B 86, 161404 (2012).

${ }^{22}$ R. Avriller and T. Frederiksen, Phys. Rev. B 86, 155411 (2012).

${ }^{23}$ A. J. White and M. Galperin, Phys. Chem. Chem. Phys. 14, 13809 (2012).

${ }^{24}$ Y. Dubi and M. Di Ventra, Rev. Mod. Phys. 83, 131 (2011).

${ }^{25}$ S. Ballmann, R. Härtle, P. B. Coto, M. Elbing, M. Mayor, M. R. Bryce, M. Thoss, and H. B. Weber, Phys. Rev. Lett. 109, 056801 (2012).

${ }^{26}$ R. Härtle, M. Butzin, O. Rubio-Pons, and M. Thoss, Phys. Rev. Lett. 107, 046802 (2011)

${ }^{27}$ K. Burke, R. Car, and R. Gebauer, Phys. Rev. Lett. 94, 146803 (2005).

${ }^{28}$ T. Frederiksen, M. Paulsson, M. Brandbyge, and A.-P. Jauho, Phys. Rev. B 75, 205413 (2007)

${ }^{29}$ W. Lee, N. Jean, and S. Sanvito, Phys. Rev. B 79, 085120 (2009).

${ }^{30}$ J. Hu, R.-X. Xu, and Y. Yan, J. Chem. Phys. 133, 101106 (2010).

${ }^{31}$ A. B. Migdal, Sov. Phys. JETP 7, 996 (1958).

${ }^{32}$ L. K. Dash, H. Ness, and R. W. Godby, Phys. Rev. B 84, 085433 (2011).

${ }^{33}$ J. Rammer, Quantum Field Theory of Non-Equilibrium States, revised edition (Cambridge University Press, 2007).

${ }^{34}$ J. M. Luttinger, Phys. Rev. 119, 1153 (1960).

${ }^{35}$ J. M. Luttinger and J. C. Ward, Phys. Rev. 118, 1417 (1960).

${ }^{36}$ G. Baym, Phys. Rev. 127, 1391 (1962).

${ }^{37}$ G. Baym and L. P. Kadanoff, Phys. Rev. 124, 287 (1961).

${ }^{38} \mathrm{~T}$. Ji, "Inelastic electron tunneling spectroscopy in molecular electronic devices from first-principles," Ph.D. dissertation (McGill University, 2010).

${ }^{39}$ H. Haug and A.-P. Jauho, Quantum Kinetics in Transport and Optics of Semiconductors, 2nd ed., Springer Series in Solid-State Sciences Vol. 123 (Springer, 2008).

${ }^{40}$ M. Büttiker, Phys. Rev. B 33, 3020 (1986).

${ }^{41}$ T. Frederiksen, M. Brandbyge, N. Lorente, and A.-P. Jauho, Phys. Rev. Lett. 93, 256601 (2004).

${ }^{42}$ A. Stan, N. E. Dahlen, and R. van Leeuwen, J. Chem. Phys. 130, 224101 (2009).

${ }^{43}$ Y. Saad, Iterative Methods for Sparse Linear Systems, 2nd ed. (Society for Industrial and Applied Mathematics, 2003).

${ }^{44}$ W. P. Su, J. R. Schrieffer, and A. J. Heeger, Phys. Rev. Lett. 42, 1698 (1979).

${ }^{45}$ M. Galperin, M. A. Ratner, A. Nitzan, and A. Troisi, Science 319, 1056 (2008) 